\title{
La gouvernance de la sécurité au sein de l'UE : un nouveau défi pour le Canada
}

\author{
Chantal Lavallée $^{1}$
}

\begin{abstract}
Since 1976, Canada and the EU developed and enlarged their relations including not only investment and trade, but also international security, environment, justice, culture, etc. During the last decade, the European Union progressively became an actor in the field of security and defence with the adoption of the European Security and Defence Policy (ESDP). ESDP is entering in the logic outside of the traditional framework analysis that conceives security as a natural input, linked to territorial defence and assumed only by the State via military means. If ESDP entered in an intergovernmental logic, it is no longer the exclusive field of the Member States, but rather the result of interactions between a broad variety of public and private actors. In this new EU security governance, Canada is bound to face a major challenge, but it's not sure that it is conscious of all the possibilities for its foreign and security policy!
\end{abstract}

Keywords: ESDP, security governance, Canada-EU relations.

\section{Résumé}

Depuis 1976, le Canada et l'UE ont développé et élargi leurs relations qui ne concernent pas seulement l'investissement et le commerce, mais aussi la sécurité internationale, l'environnement, la justice, la culture, etc. Au cours de la dernière décennie, l'UE est devenue un acteur dans le domaine de la sécurité et la défense avec l'adoption de la Politique européenne de sécurité et de défense (PESD) ce qui a eu un impact sur les relations UE-Canada. Il faut dire que la portée de la PESD dépasse le cadre d'analyse traditionnel qui conçoit la sécurité comme une donnée naturelle, liée à la défense territoriale et assumée seulement par l'Etat à l'aide de moyens essentiellement militaires. En effet, si la PESD s'inscrit officiellement dans une logique intergouvernementale, elle ne concerne pas que les Etats, mais elle est plutôt le résultat d'interactions entre une grande variété d'acteurs publics et privés. Dans cette nouvelle perspective de la gouvernance de la sécurité au sein de l'UE, le Canada est confronté à un défi de taille, mais encore faudrait-il savoir s'il est conscient de toutes les possibilités pour sa politique étrangère et de sécurité !

Mots clés : gouvernance de la sécurité,PESD, relations Canada-UE.

\footnotetext{
${ }^{1}$ Doctorante rattachée au Centre d'études des politiques étrangères et de sécurité (CEPES), chercheuse associée à la Chaire de recherche du Canada en politiques étrangère et de défense canadiennes et chargée de cours au Département de science politique de l'Université du Québec à Montréal (UQÀM).
} 


\section{Introduction}

Le Canada et l'Union européenne (UE) entretiennent, depuis 1976 et l'accord-cadre de coopération économique et commerciale ${ }^{2}$, des relations qui, au-delà du commerce et de l'investissement, ont été élargies au fil du temps à de nombreux domaines tels que la sécurité internationale, l'environnement, la justice, les migrations, l'éducation, la culture, etc. Les relations entre le Canada et l'UE ont ainsi su s'adapter à la nouvelle donne internationale. ${ }^{3}$ Par ailleurs, l'intégration européenne a, elle aussi, bien progressé, particulièrement au cours de la dernière décennie. L'Union européenne (UE) s'est, entre autres, progressivement affirmée sur la scène internationale, en se donnant les moyens d'assumer ses responsabilités en matière de politique étrangère, de sécurité et de défense. L'UE est ainsi passée de la Coopération politique européenne $(\mathrm{CPE})$, forum de discussion informelle en matière de politique étrangère, à l'adoption de politiques visant à coordonner son action également dans le domaine de la sécurité et de la défense.

La Politique européenne de sécurité et de défense (PESD) fait partie intégrante de la Politique étrangère et de sécurité commune (PESC), et s'inscrit donc dans le second pilier de l'UE qui répond officiellement à une logique intergouvernementale. Cependant, Gisela Müller-BrandeckBocquet maintient que le processus décisionnel est beaucoup plus complexe et subtil qu'il n'y paraît. Elle traduit cette complexité par la présence de quatre principes à l'œuvre au sein de la PESD, soit l'intergouvernementalisme, la souveraineté partagée, la «bruxellisation » et le supranationalisme. Ce qui l'amène à conclure à l'émergence d'une troisième voie entre l'intergouvernementalisme et le supranationalisme. ${ }^{4}$ Le phénomène $\mathrm{d}$ ' «interpilarisation ${ }^{5}$, soit d'une coopération entre les trois piliers sur lesquels repose l'UE, qui est de plus en plus à l'œuvre dans la gestion de cette politique, a sûrement contribué à cette transformation. Il y a, en effet, plusieurs acteurs issus des différentes institutions européennes qui interviennent et interagissent en matière de PESD. En décloisonnant l'analyse de cette politique atypique à sa seule dimension intergouvernementale, Müller-Brandeck-Bocquet ouvre un débat fort intéressant bien qu'elle ne s'attarde qu'aux acteurs officiels. Il apparaît pourtant invraisemblable que les acteurs nationaux comme les acteurs européens travaillent en vase clos et qu'ils soient isolés des influences extérieures. Nous assistons plutôt à l'émergence d'une nouvelle forme de gouvernance au sein de l'UE, en ce qui concerne la gestion de la sécurité. Une situation nouvelle qui se présente également comme un défi pour les pays tiers tel que le Canada. A ce propos, nous pouvons questionner le positionnement du Canada à l'égard de l'Europe. Prend-t-il vraiment en compte la nouvelle dynamique au sein de l'UE et les possibilités qui s'offrent à lui en matière de politique étrangère, de sécurité et de défense dans l'élaboration de sa position vis-à-vis de l'Europe?

Dans le cadre de cet article, j'essayerai, dans un premier temps, de montrer comment se traduit cette gouvernance européenne de la sécurité, qui sont les acteurs, quels sont les enjeux et les

\footnotetext{
${ }^{2}$ A ce moment là, il s'agit du premier accord de coopération de la Communauté avec un pays industrialisé.

${ }^{3}$ L'accord-cadre de 1976 demeure la principale base juridique des relations formelles qu'entretiennent l'UE et le Canada, mais des déclarations politiques ont été adoptées en 1990 et en 1996, de manière à faciliter la coopération dans un plus large éventail de domaines.

${ }^{4}$ Gisela Müller-Brandeck-Bocquet, «The New CFSP and ESDP Decision-Making System of the European Union », European Foreign Affairs Review, vol. 7, no. 3 (2002), pp. 257-282.

${ }^{5}$ Simon Duke, «CESDP: Nice's Overtrumped Success? », European Foreign Affairs Review, vol. 6, no. 2 (2001), p. 174.
} 
processus qui l'animent? Dans un deuxième temps, j'évaluerai l'impact de cette nouvelle donne européenne sur les relations transatlantiques, particulièrement entre le Canada et l'UE.

\section{La gouvernance de la sécurité au sein de l'UE}

Un nouveau type de gouvernance a pris forme au sein de $1^{\prime} \mathrm{UE}^{6}$, non pas en termes de niveaux hiérarchiques, où l'État est nécessaire au sommet, mais plutôt comme un système d'interactions entre différents réseaux d'acteurs. Force est d'admettre que la décision finale est peut-être prise par les acteurs étatiques, mais la compréhension de la décision passe par l'étude du processus qui la précède. Dès lors que nous parlons de gouvernance de la sécurité, il importe d'accorder une attention particulière aux acteurs publics et privés, à leur rôle, à leur trajectoire socioprofessionnel, à leurs interactions, aux possibles compétitions institutionnelles ou luttes de positionnement afin d'éviter que l'étude ne soit trop descriptive. Cette approche sociologique est d'autant plus utile qu'elle révèle de nouvelles pratiques de sécurité tant au niveau des acteurs étatiques que non étatiques.

\section{Les acteurs étatiques et européens au sein d'un processus décisionnel de plus en plus ouvert}

Les prises de position des chefs d'État et de gouvernement au sein du Conseil européen et des ministres des Affaires étrangères au sein du Conseil, sont le résultat d'interactions entre un ensemble d'individus. Considérant que les ministres de la Défense ne se réunissent pas au sein du Conseil, mais seulement dans le cadre de rencontres informelles, il y a de nombreuses interactions entre les ministères de la Défense et des Affaires étrangères qui ne sont souvent pas dépourvues de compétitions bureaucratiques. S'inscrivant dans des contextes historiques spécifiques, la relation entre le politique et le militaire, voire ce rapport de force, varie d'un pays à l'autre et, dans certains cas, a un impact direct sur la prise de décision. ${ }^{7}$ Également, ces prises de position sont issues des interactions entre les homologues des ministères des États membres de l'UE. Au niveau des ministères, de nombreux échanges de fonctionnaires entre les différents pays de l'UE jouent aussi un rôle dans le rapprochement des positions, à tout le moins, ils favorisent une meilleure compréhension des visions de chacune des administrations. ${ }^{8}$ Par exemple, la fonctionnaire rattachée à la Délégation des Affaires stratégiques (DAS) du Ministère français de la Défense et qui fut envoyée en échange pendant trois ans, au Ministère de la Défense britannique, y a été nommée l'officier responsable de la PESD, témoignant du sérieux et de la portée de ces échanges. ${ }^{9}$

Les positions du Conseil européen et du Conseil des ministres résultent aussi de négociations intenses entre les fonctionnaires travaillant au sein des ambassades, mais surtout, et de plus en plus, au sein des Représentations permanentes des États membres auprès de l'UE (RP). En effet,

\footnotetext{
${ }^{6}$ Mark Webber et al., «The governance of European Security », Review of International Studies, vol. 30, no. 1 (2004), pp. 3-26. Dans cet article, nous pouvons lire une section sur la gouvernance de la sécurité avec la PESD, mais la perspective est plutôt descriptive que théorique. Je pense que nous pouvons pousser la réflexion plus en profondeur sur les conséquences d'une telle gouvernance.

7 Frédéric Mérand, «Social Representations in the European Security and Defence Policy », Cooperation and Conflict, vol. 41, no. 2 (2006), pp. 131-152.

${ }^{8}$ Jolyon Howorth, «Discourse, Ideas and Epistemic Communities in European Security and Defence Policy», West European Politics, vol. 27, no. 2 (2004), p. 220.

${ }^{9}$ Entretien réalisé au Ministère de la Défense britannique, en mai 2005.
} 
le centre d'action de l'UE se trouve indéniablement à Bruxelles. Situées au cœur de l'activité européenne, les représentations permanentes favorisent les rencontres sur une base régulière entre les représentants des pays membres. La socialisation y est particulièrement forte entre les homologues des représentations qui se connaissent très bien et se côtoient, aussi souvent que nécessaire. Dans le cas des homologues français et britanniques, il n'est pas rare qu'ils se retrouvent dans un cadre informel, chacun muni de leur feuille de route afin de trouver un compromis et de faire converger les directives venant de leur gouvernement respectif. ${ }^{10}$ Il faut préciser que les représentations permanentes abritent toute une équipe de fonctionnaires travaillant sur les questions de sécurité et de défense, sous la direction de l'ambassadeur au Comité politique et de sécurité (COPS) et du représentant au Comité militaire de l'UE.

Les représentations permanentes jouent un rôle incontournable, d'autant plus qu'elles sont connectées aux réseaux d'acteurs transnationaux. En effet, l'État n'est plus le seul acteur à intervenir dans la gestion de la sécurité. Michel Barnier l'a bien compris, ancien commissaire européen, une fois nommé ministre français des Affaires étrangères, il a voulu remédier à la perte d'influence de la France auprès de l'UE. ${ }^{11}$ Pour lui, il est clair à ce moment que «si Paris ne favorise pas très vite la création de think tanks indépendants, mais ouverts aux idées françaises, la France va perdre toute capacité de persuasion en Europe. La représentation permanente de la France auprès de l'UE a depuis une attachée chargée des relations avec les think tanks... ${ }^{12}$ Nous retrouvons, à ce propos, sur son site Internet, une section intitulée «Cercles de réflexion/Think tanks». «Un dispositif de veille des activités des principaux think tanks traitant des questions européennes ainsi qu'une politique de contact ${ }^{13}$ a été mis en place. Des consultants, des acteurs issus d'organisations non gouvernementales (ONG) et de think tanks ont ainsi des contacts réguliers avec les représentations permanentes qui, selon eux, jouent un rôle important dans la définition de la PESD et sont ainsi des lieux clés dans leur stratégie d'influence. $^{14}$

De même, j'y reviendrai, mais ces acteurs non étatiques entretiennent des liens étroits avec les différentes institutions européennes. La PESD est dotée de structures qui lui sont propres, au sein de l'UE, permettant entre autres à l'UE de mener des missions civiles et militaires. Il s'agit du COPS, du Comité militaire de l'UE (CMUE) et de l'État-major de l'UE (EMUE). Le SITCEN et la Direction générale-E du Secrétariat viennent aussi appuyer le travail du Secrétaire général du Conseil Javier Solana qui, depuis 1999, occupe aussi le poste de Haut Représentant pour la PESC (HR). Communément appelé Monsieur PESC, Javier Solana, en plus de personnifier l'UE sur la scène internationale, joue un rôle non négligeable de négociateur, de facilitateur, de médiateur et détient un certain degré d'autonomie. Pour ce qui est des autres institutions européennes, la

\footnotetext{
${ }^{10}$ Entretiens réalisés au sein des représentations permanentes britannique et française auprès de l'UE, en juin 2005.

11 «Michel Barnier estime néanmoins que la France doit réagir au changement d'environnement européen et mondial en revoyant son mode d'action. » rapportait Henri de Bresson dans son article « Michel Barnier : la diplomatie de la France passe par l'Europe », Le Monde (En ligne), 27 août 2004, www.lemonde.fr/web/imprimer_article/0,1-0@23214,36-376747,0.html

${ }^{12}$ Stephen Boucher et Martine Royo, Les think tanks. Cerveaux de la guerre des idées, Paris, Éditions du Félin, 2006, p. 57.

${ }^{13}$ La Représentation permanente de la France auprès de l'UE, Cercles de réflexion/think tanks, (En ligne) http://www.rpfrance.eu/article/imprim.php3?id_article=221 (page consultée le 7 septembre 2006).

${ }^{14}$ Entretiens réalisés avec des acteurs issus de think tanks et d'ONG, en juillet 2006.
} 
Commission fait partie de la troïka ${ }^{15}$ et peut ainsi participer aux rencontres du Conseil européen et du Conseil des ministres concernant la PESC/PESD. Dans l'esprit de la stratégie européenne de sécurité, c'est un véritable exercice de coordination qui se met en branle entre les Directions générales du Commerce, de l'Aide humanitaire, du Développement, de l'Élargissement, des Relations extérieures et des délégations de la Commission à travers le monde; sans négliger le rôle de gestionnaire de la Commission en matière du budget PESC/PESD, concernant les aspects administratifs et civils. Le Parlement européen, pour sa part, s'il joue un rôle marginal, doit néanmoins être régulièrement informé et consulté par la présidence de l'UE, le HR et la Commission sur l'évolution de la PESC/PESD. Il peut adresser des questions au Conseil. Au sein du Parlement, la Commission des Affaires étrangères, assistée par la sous-commission «sécurité et défense », est ainsi chargée de suivre les dossiers de la PESC et de la PESD. Cependant, le Parlement n'exerce aucun pouvoir direct en la matière et son droit de recommandation est plus politique que juridique. Le Parlement est, par ailleurs, directement concerné au niveau des sommes allouées au budget de la PESC/PESD pour les aspects administratifs et civils.

Bref, voilà autant de points d'entrées et de lieux d'influence pour les acteurs non étatiques qui souhaitent participer au débat et peser sur les orientations données à la PESD.

\section{L'émergence d'acteurs non étatiques au sein d'une nouvelle gouvernance, celle de la sécurité européenne}

Il est vrai que le phénomène est encore très récent en Europe, surtout si nous le comparons avec ce qui se passe aux États-Unis, mais de plus en plus de think tanks, de consultants et d'ONG vont s'installer à Bruxelles; la capitale européenne est devenue un bon endroit pour développer et entretenir des contacts. ${ }^{16}$ Le rôle grandissant de l'UE sur la scène internationale, n'est sûrement pas étranger à l'émergence de ces acteurs. La portée de l'action de ces acteurs est générale et globale, une conséquence de l'augmentation du pouvoir, du champ de compétences et du rôle de l'UE dans le monde. Ainsi, les think tanks et les ONG cherchent à jouer un rôle auprès de l'UE, à avoir un impact sur ses politiques et surtout, à avoir accès au débat européen. ${ }^{17} \mathrm{Qu}$ 'ils mènent des études pour les gouvernements ou les institutions européennes, ils possèdent une expertise et cherchent, de plus en plus, à influencer quelque soit le domaine, y compris celui de la sécurité et la défense. Le changement d'attitude des gouvernements qui, à la suite de la fin de la Guerre froide, ont dû composer avec des coupures budgétaires importantes et le besoin de garder une maîtrise des dossiers, a ouvert le processus quant à l'UE, sa structure même bouscule les paramètres traditionnels de la gestion des dossiers, y compris celui de la sécurité. Il faut, dès lors, repenser la gestion de la sécurité dans une perspective de gouvernance au sein de laquelle se déroulent des interactions entre acteurs publics et privés, organisant leurs intérêts communs et divergents.

Dans l'étude des acteurs de la société civile en Europe, il faut souligner un cas d'exception, un think tank proprement européen. En effet, l'UE est la seule organisation régionale à avoir son

\footnotetext{
${ }^{15}$ La troïka comprend la présidence en exercice de l'UE, le Haut représentant pour la PESC et un représentant de la Commission européenne. La troïka est surtout active sur la scène internationale.

${ }^{16}$ Comme en témoigne Annuaire des Think Tanks traitant des questions européennes tel que présenté sur le site de la Représentation permanente de la France auprès de l'UE, http://www.rpfrance.eu/article.php3?id_article=218

${ }^{17}$ Remarques faites par plusieurs acteurs qui travaillent à Bruxelles et que j'ai rencontrés en entrevue, en juillet 2006.
} 
propre think tank, une véritable boîte à penser sur l'Europe. Il s'agit de l'Institut d'études de sécurité (IES) de l'Union européenne, hérité de l'Union de l'Europe occidentale (UEO). Cet institut de recherche invite, en rotation, des chercheurs venant des différents pays membres de l'UE. Il est d'ailleurs surprenant que la première institution du genre se concentre sur les enjeux rattachés à la sécurité européenne alors que l'UE traite de milliers d'autres sujets. «L'IES contribue au développement de la PESC en assurant trois fonctions : la recherche et le débat sur les questions de sécurité pertinentes pour l'Union; l'analyse prospective pour le Conseil et le Haut Représentant de l'UE; le développement d'un dialogue transatlantique sur l'ensemble des questions de sécurité entre tous les pays européens, le Canada et les États-Unis. ${ }^{18}$ Le statut de l'Institut d'études de sécurité donne aux chercheurs qui y travaillent un accès privilégié aux fonctionnaires et aux dirigeants européens, ce qui n'est pas donné à tous les think tanks. Malgré l'aspect novateur entourant la mise en place de cette agence, il y a des limites à l'action de l'IES puisqu'elle n'a pas tout à fait le même rôle qu'un think tank «traditionnel ». En effet, les chercheurs n'y ont pas nécessairement la même marge de manœuvre bien qu'officiellement indépendante, l'IES n'en demeure pas moins une agence européenne.

Ceci étant dit, dans ce nouveau contexte européen, des réseaux transnationaux d'acteurs s'organisent de manière formelle et informelle. Il est indéniable que «la disponibilité des fonds européens pour des think tanks qui établissent des partenariats de recherche avec d'autres think tanks et organisations à travers l'Union a fait progresser la collaboration dans ce secteur. » ${ }^{19}$ Par exemple, la Commission européenne finance The Conflict Prevention Partnership qui regroupe International Crisis Group, International Alert, European Policy Centre et European Peacebuilding Office. Ce partenariat vise, entre autres, à renforcer les capacités de l'UE et de ses membres dans la prévention de conflit, la gestion de crise et la reconstruction de la paix. ${ }^{20}$ Un exemple de l'étroite collaboration entre ces acteurs issue de ce partenariat, Gareth Evans, président de l'International Crisis Group, est aussi membre du conseil de l'European Policy Centre (EPC). ${ }^{21}$ À côté de ces partenariats officiels, nous retrouvons aussi des coopérations informelles entre thinks tanks, ONG et consultants. ${ }^{22}$ Ce qui confirme qu'il n'y a pas un réseau, mais bien des réseaux d'acteurs publics et privés qui se chevauchent et qui s'entrecroisent.

Les think tanks peuvent aussi servir de moyen d'influence pour les États membres, j'ai déjà noté le cas de la France qui souhaite relancer sa capacité d'influence à Bruxelles, en encourageant l'émergence de think tanks, mais elle n'est pas la seule. «Londres assure son influence auprès des institutions européennes notamment par l'envoi de diplomates extrêmement bien formés aux

\footnotetext{
18 Institut d'études de sécurité de l'Union européenne. Sur l'IES, (En ligne). http://www.iss.europa.eu/ (page consultée le 28 février 2007)

19 Stephen Boucher (dir.), L'Europe et ses think tanks : un potentiel inaccompli, Paris, Notre Europe (En ligne), 2004, p. 12. http://www.notre-europe.asso.fr/article.php3?id_article=537\&lang=fr

${ }^{20}$ Conflict Prevention Partnership, About the Partnership, (En ligne). http://www.conflictprevention.net/index.cfm?if=99 (page consultée le 22 juin 2006)

21 European Policy Centre, Governance: Advisory Council, (En ligne). http://www.theepc.be/en/ae2.asp? (page consultée le 28 juin 2006)

${ }^{22}$ Entretiens réalisés avec différents acteurs issus de think tanks, d'ONG et de consultants pour des entreprises privées, en 2006 et en 2007.
} 
questions européennes. Les think tanks sont (aussi) un relais de persuasion de la diplomatie intellectuelle britannique efficace. ${ }^{23}$

Les think tanks comme les ONG gagnent également en importance et en crédibilité puisqu'ils deviennent des lieux de formation, de véritables « réservoir(s) d'experts ${ }^{24}$. Toutes les personnes qui y travaillent sont susceptibles d'être éventuellement embauchées comme fonctionnaire au niveau national ou au niveau européen. Ceci vaut aussi dans l'autre sens, il n'est pas rare de voir d'anciens fonctionnaires ou des militaires à la retraite poursuivre leur carrière au sein de think tanks, d'ONG ou à titre de consultant, mettant ainsi à profit leur expertise acquise sur le terrain et surtout leur réseau de contacts. Désormais, ce sont des acteurs connus qui ont donc un lien de confiance avec les acteurs étatiques, grâce à leur passé professionnel, mais surtout grâce à leur expertise. Il n'y a pas de surprise, nous sommes bien en présence de réseaux d'acteurs, où les frontières sont poreuses. Une étude des trajectoires de ces acteurs s'impose donc pour saisir les dynamiques internes. En consultant la liste des membres des conseils de think tanks européens et d'ONG, il est frappant de constater que la majorité de ces membres sont d'anciens fonctionnaires européens ayant occupé des postes de haut niveau, d'anciens premiers ministres et ministres issus des pays membres. Par exemple, Christopher Patten qui a une longue carrière en tant que diplomate britannique, fut le commissaire responsable de la DG-RELEX de 1999 à 2004 et est, à présent, coprésident de l'International Crisis group et son parcours est loin d'être atypique.

S'il demeure difficile, voire impossible, de mesurer l'influence réelle de ces acteurs sur l'élaboration de la PESD, nous ne pouvons pas la nier pour autant. Il faut consulter les sites Internet de think tanks, d'ONG, de consultants pour constater l'intérêt manifesté à l'égard de cette politique atypique. En plus, de la tenue d'événements d'envergure, nous y retrouvons des rapports dans lesquels des recommandations sont formulées à l'attention des fonctionnaires européens et des dirigeants des États membres de l'UE.

D'autres acteurs cherchent aussi à influencer et à se positionner, il s'agit de consultants auprès d'entreprises privées ou de représentants des entreprises elles-mêmes qui répondent aux appels d'offres des institutions européennes, de l'Agence européenne de défense ou des gouvernements des États membres. Ces acteurs n'évoluent pas seuls, indépendamment les uns des autres, mais s'inserts plutôt dans les différents réseaux de conseil avec des liens très forts autant intellectuels que commerciaux. Dans ces réseaux, nous retrouvons donc tant des think tanks, des ONG que des consultants ou des entreprises privées. Dès lors, il n’est pas rare de voir des représentants d'entreprises participer aux activités organisées par des think tanks ou des ONG et publier des articles sur les sites Internet ou dans les publications de ces organisations, autant de façon pour les acteurs privés de diffuser leurs idées et leurs visions de la sécurité et de la défense en Europe par le biais des différents réseaux à leur disposition.

\section{Les relations canado-européennes dans une nouvelle géométrie}

Il est, dès lors, pertinent de s'interroger sur l'impact de cette nouvelle gouvernance au sein de l'UE sur les relations transatlantiques, particulièrement entre l'UE et le Canada. Est-ce que le

\footnotetext{
${ }^{23}$ Stephen Boucher et Martine Royo, op.cit., pp. 66-67.

${ }^{24}$ Stephen Boucher (dir.), L'Europe et ses think tanks... op.cit., p. 37.
} 
Canada prend suffisamment en compte la transformation opérée du côté européen en matière de sécurité dans la gestion de ses relations avec l'UE? Le gouvernement canadien s'est-il positionné de façon stratégique dans ce nouveau contexte? Utilise-t-il tous les moyens à sa disposition pour se faire entendre auprès de Bruxelles?

L'UE et le Canada entretiennent depuis longtemps des relations de manière formelle qui touchent, entre autres, la sécurité internationale. Le dialogue de haut niveau est assuré par des Sommets et des rencontres ministérielles, mais aussi par des contacts entre les directeurs politiques et entre les diplomates. ${ }^{25}$ A titre d'exemple, dans le domaine qui nous intéresse pour cet article, lors du sommet UE-Canada, du 24 novembre 2005, un accord établissant un cadre pour la participation du Canada aux opérations de gestion de crise menées par l'UE, a ainsi été signé. Le Canada a d'ailleurs participé à plusieurs missions européennes, depuis 2003, mais la base de cette collaboration fut jusque-là $a d$ hoc bien que le gouvernement canadien a toujours souhaité un cadre juridique pour préciser les modalités de sa participation à ces missions, espérant y jouer un rôle accru. ${ }^{26}$ Comment le gouvernement canadien est-il parvenu à un tel accord?

Au-delà des sommets, des rencontres et réunions officielles entre les représentants de l'UE et du Canada, il y a donc d'autres facettes de ces relations à prendre en compte afin de saisir comment le gouvernement canadien parvient à faire entendre sa position. A titre de bailleur de fonds ou de membre de think tanks et d'ONG, nous retrouvons aussi des ministères des Affaires étrangères ou des ressortissants d'États tiers. En effet, si les think tanks peuvent servir de moyen d'influence auprès de l'UE pour les États membres, les pays tiers ont bien compris qu'ils pouvaient eux aussi y recourir, d'autant plus qu'ils ne sont pas membres de l'UE. En s'associant avec ces acteurs, les États tiers cherchent à bénéficier des réseaux de contacts de ces organisations pour avoir accès aux décideurs européens, aux analyses des experts ou tout simplement pour suivre et participer aux débats au sein de l'UE, y compris sur les questions de sécurité et de défense. Ces diverses organisations deviennent ainsi, pour la plupart, de véritables agences de réseautage, d'ailleurs ces acteurs se présentent souvent comme des joueurs clés à la croisée des chemins, à l'intersection entre les dirigeants européens et la société civile. Dans ce nouveau contexte, le Canada ne fait pas exception.

Ainsi, l'Agence canadienne de développement international (ACDI), le Centre canadien de recherches pour le développement international et le ministère des Affaires étrangères et du Commerce international du Canada contribuent au financement de l'International Crisis Group (ICG). L'ICG, dont j'ai déjà parlé, est une ONG à but non lucratif qui fait partie de différents réseaux de recherche et d'influence et qui travaille à la prévention et la résolution des conflits, grâce à des envoyés sur le terrain et une équipe de représentants, entretenant des contacts étroits avec les décideurs. Cette ONG ne se concentre pas seulement sur l'UE, mais a une équipe à Bruxelles et a réalisé différents rapports incluant des recommandations s'adressant à l'UE et concernant son rôle sur la scène internationale. À noter que deux Canadiens se retrouvent dans le

25 Conseil de l'UE, Sommet UE-Canada, Ottawa, 18 mars 2004, 7431/04 (Presse 83), pp. 5-6. http://www.consilium.europa.eu/ueDocs/cms_Data/docs/pressData/fr/er/79509.pdf

${ }^{26}$ Conseil de l'UE, Javier Solana signs EU-Canada Agreement establishing a framework for the participation of Canada in EU crisis management operations, Bruxelles, 24 novembre 2005, S381/05, http://www.consilium.europa.eu/ueDocs/cms_Data/docs/pressData/en/declarations/87119.pdf 
comité exécutif de l'ICG, soit Frank Giustra d'Endeavour Financial et Kim Campbell qui fut brièvement première ministre du Canada, en 1993. Par ailleurs, concernant les think tanks, Jeremy Kinsman, l'ancien ambassadeur à la Mission du Canada auprès de l'UE, fut pendant son mandat à Bruxelles un membre du Conseil de l' European Policy Centre (EPC), un think tank bruxellois. ${ }^{27}$ Un rôle que son successeur Ross Hornby, en poste depuis août 2006, n'a cependant pas conservé.

Voilà qui m'amène à parler de la Mission du Canada auprès de l'UE qu'on peut comparer aux représentations permanentes des États membres auprès de l'UE, dans la mesure où elle devient aussi un lieu incontournable de socialisation pour les différents acteurs impliqués dans les relations entre le Canada et l'UE. D'abord, à l'intention des diplomates canadiens provenant des diverses ambassades en Europe et de ceux basés à Ottawa, elle organise des séminaires sur le fonctionnement des institutions européennes et le rôle de l'UE en général. ${ }^{28}$ Egalement, un fonctionnaire est assigné aux dossiers concernant la sécurité et la défense de l'UE et les relations avec le Canada dans ce domaine. Il est régulièrement en contact d'une part avec la Direction de l'UE au sein du ministère des Affaires étrangères et du Commerce international (MAECI), à Ottawa et de l'autre, avec tous les acteurs nationaux, européens et transnationaux susceptibles de l'aider dans son travail. ${ }^{29}$ La Mission du Canada auprès de l'UE entretient des relations particulières avec deux think tanks situés à Bruxelles, le Centre for European Policy Studies (CEPS) et l'EPC. Par exemple, lors de la visite de Jean Charest, à Bruxelles, en mars 2005, le premier ministre québécois a participé avec Jeremy Kinsman et des représentants du Conseil et de la Commission européenne à une conférence intitulée «Quebec/Canada and the EU: a Growing and Strategic Transatlantic Relation » et organisée en collaboration et dans les locaux du CEPS. ${ }^{30}$ De même, le 5 décembre 2006, la Mission du Canada en collaboration avec l'EPC a réuni un groupe de discussion de haut niveau sur «Le rôle de la sécurité humaine dans la politique étrangère : quelles leçons pour l'UE?». Le panel comptait, entre autres, le nouvel ambassadeur Ross Hornby, Mary Kaldor qui a présidé le groupe d'étude qui a rédigé «A Human Security Doctrine for Europe » pour le HR et Richard Wright, directeur de la Plateforme de crise PESC à la Commission européenne. ${ }^{31}$

La Mission du Canada compte aussi parmi ses principaux partenaires le Forum sur le Commerce Canada-Europe (FORCCE) qui «a été créé en 1999 par différentes sociétés européennes et canadiennes cherchant à répondre au besoin d'un dialogue efficace entre le Canada et l'Europe sur les enjeux d'affaires, ouvrant la voie à une plus grande coopération transatlantique à tous les niveaux de la relation Canada-UE. ${ }^{32}$ Dans cette perspective, il est intéressant de noter qu' un des quatre groupes de travail du FORCCE, celui concernant la Recherche et le Développement, est

\footnotetext{
27 European Policy Centre, Governance: Advisory Council, (En ligne). http://www.theepc.be/en/ae2.asp? (page consultée le 28 juin 2006)

${ }^{28}$ Mission du Canada auprès de l'UE, Événements Canada-UE, (En ligne). http://www.dfait-maeci.gc.ca/canadaeuropa/eu/can_eu events-fr.asp (page consultée le 1er mars 2007)

${ }^{29}$ Entretien réalisé à la Mission du Canada auprès de l'UE, Bruxelles, mars 2005.

30 Jean Charest, Jeremy Kinsman, Jim Cloos et Richard Wright, «Quebec/Canada and the EU: a Growing and Strategic Transatlantic Relation», CEPS, 3 mars 2005, http://www.ceps.be/Event.php?event_id=102

${ }^{31}$ «Le rôle de la sécurité humaine dans la politique étrangère : quelles leçons pour l’UE? », EPC, décembre 2006, http://www.epc.eu/en/er.asp?TYP=ER\&LV=293\&see=y\&t=2\&PG=ER/EN/detail\&l=\&AI=660

32 Mission du Canada auprès de l'UE, Nos partenaires, (En ligne), http://www.dfait-maeci.gc.ca/canadaeuropa/eu/partners-fr.asp (page consultée le 1er mars 2007)
} 
présidé par Bombardier et EADS. ${ }^{33}$ Pourtant, Bombardier demeure peu présent dans les cercles de réflexion en Europe, contrairement à d'autres compagnies du secteur tel que Boeing. Dans un autre ordre d'idées, il y a également l'Association d'études sur la Communauté européenneCanada (ECSA-C) qui veille au développement d'échanges entre des chercheurs canadiens et européens sur leur activité de recherche portant sur l'UE, comme en témoigne la Conférence transatlantique du Réseau canadien des jeunes chercheurs en études européennes, intitulée « L'Union européenne et le Canada : défis communs et leurs réponses politiques » qui a eu lieu à l'Institut Universitaire Européen (IUE), à Florence, en Italie, le 27 avril 2007 et qui est à l'origine de ce numéro spécial de la Revue des affaires européennes et russes. L'ECSA-C entretient des contacts étroits avec la Délégation de l'UE à Ottawa et le ministère canadien des Affaires étrangères et du Commerce international et se présente comme une plateforme de discussion entre les décideurs, les chercheurs, les médias et les entreprises. ${ }^{34}$ Nous pouvons bien sûr nous interroger sur l'influence réelle de ces organisations, néanmoins nous assistons à une multiplication de lieux de débat, d'acteurs et de moyens à la disposition du Canada, autant des acteurs étatiques que de ceux de la société civile pour faire entendre leur position et leurs attentes dans leurs relations avec l'UE.

Il a été question plus haut de la visite du premier ministre québécois Jean Charest, à Bruxelles, il ne faut donc pas oublier la présence de la Délégation générale du Québec à Bruxelles qui, depuis 1972, a pour mandat d'assurer le rayonnement du Québec et la promotion de ses intérêts, entre autres, au sein des institutions européennes avec le Service des affaires européennes qui veille « à ce que le Québec constitue un interlocuteur actif dans le cadre de la coopération entre le Canada et l'Union européenne. ${ }^{35}$ Un autre acteur qu'il faut donc également prendre en considération dans les relations Canada-UE sous l'angle essentiellement de la politique étrangère et non pas de la sécurité ni de la défense qui nous intéresse dans cet article puisqu'elles ne relèvent pas des compétences provinciales. La Délégation générale du Québec organise aussi des séminaires de formations et des missions pour des personnalités politiques, des représentants d'institutions ou organismes québécois, comme ce fut le cas lors de la visite de Jean Charest, en 2005. D'ailleurs, la Délégation générale du Québec possède un réseau de contacts impressionnant qui en surprend plusieurs, à commencer par les fonctionnaires travaillant à la Mission du Canada. Il ne faut pas pour autant en déduire que la Mission du Canada et la Délégation générale du Québec sont en compétition, au contraire, elles sont amenées à coopérer dans la tenue d'événements. ${ }^{36}$

Voilà qui ouvre un débat intéressant sur une nouvelle façon d'aborder les relations entre le Canada et l'UE. Si ces exemples laissent croire à un positionnement stratégique du Canada à l'égard de la nouvelle réalité européenne en matière de politique étrangère, de sécurité et de défense, je m'interroge néanmoins sur l'absence de représentants canadiens, soit à titre de partenaires ou à titre de membre au sein du conseil d'ONG et de think tanks stratégiques ${ }^{37}$, où

33 Forum sur le Commerce Canada-Europe, Structure et fonctionnement, (En ligne), http://www.canadaeurope.org/fr/AboutUs/operatingStructure.htm (page consultée le 2 mars 2007)

34 Association d'études sur la Communauté européenne-Canada, About Us (En ligne), http://web.uvic.ca/ecsac/about.html (page consultée le 5 avril 2006)

35 La Délégation générale du Québec à Bruxelles, Nos Services (En ligne), http://www.mri.gouv.qc.ca/bruxelles/fr/delegation/qui_sommes_nous/services.asp, (page consultée le 3 mars 2007)

${ }^{36}$ Entrevue réalisée à la Mission du Canada auprès de l'UE, Bruxelles, mars 2005.

37 À titre d'exemple, Friends of Europel Les Amis de l'Europe qui se veut également un lieu de débat sur l'avenir de l'Europe, mais a priori pas sur la défense, mettant en contact des acteurs publics et privés. Entre autres activités, il 
bon nombre de représentants de pays tiers sont pourtant présents, à commencer par ceux provenant des États-Unis. De même, le Canada est officiellement absent du Security and Defence Agenda (SAD) qui a succédé au New Defence Agenda, en février 2006. En effet, si des représentants de la mission canadienne participent régulièrement aux activités du SAD, il n'y a pas de coopération formelle ou institutionnalisée. Pourtant, le SAD «se présente lui-même comme le seul forum régulier à Bruxelles, où l'OTAN, l'UE, les représentants d'entreprises et les universitaires, les personnalités politiques et les journalistes peuvent discuter du futur des politiques de sécurité et de défense européennes et transatlantiques. Le SAD sert aussi de centre de réseautage de think tanks liés à la défense à travers l'Europe. ${ }^{38}$ Il est présidé par le Secrétaire général de l'OTAN Jaap de Hoop Scheffer, par le HR Javier Solana, le Commissaire pour la Justice, Liberté et Sécurité Franco Frattini et la Commissaire pour les Relations extérieures Benita Ferrero-Waldner. Le SAD est lié au Forum Europe qui, depuis 1989, se veut également une plateforme de débat entre acteurs publics et privés. ${ }^{39}$ Dans le même ordre d'idées, les deux derniers séminaires de l'IES, qui veille, entre autres, au développement d'un dialogue transatlantique sur l'ensemble des questions de sécurité entre tous les pays européens, le Canada et les États-Unis, ont portés essentiellement sur les relations entre l'UE et les États-Unis. Il est vrai que le contexte international des dernières années peut expliquer en partie la situation, mais il n'en demeure pas moins que le Canada se fait plutôt discret et qu'il pourrait jouer un rôle plus actif, pour élargir le dialogue transatlantique aussi à la réalité canadienne qui n'est pas nécessairement celle des États-Unis. Si les représentants canadiens sont moins présents dans les réseaux de la société civile en Europe, ils privilégient peut-être davantage les contacts avec les acteurs officiels, de manière informelle, mais directement avec les personnes qui les intéressent. Peut-être ont-ils la conviction que c'est de cette façon qu'ils ont le plus grand impact et qu'ils obtiennent satisfaction?

Au-delà des acteurs étatiques canadiens, ce sont aussi les entreprises privées, les think tanks et les ONG canadiennes qui doivent se positionner dans ce nouveau paysage. La distinction entre ces acteurs s'impose en l'absence de concertation formelle, il demeure difficile de parler de la position du Canada comme d'un tout homogène. Puisque nous parlons des relations transatlantiques, il est frappant de voir des représentants d'entreprises privées, de think tanks américains occuper des postes stratégiques dans des think tanks et des ONG concernés par les questions européennes, voire installer une antenne de leur organisation à Bruxelles, particulièrement en matière de politique étrangère, de sécurité et de défense. Est-ce dû à des stratégies, des intérêts ou des budgets différents si les acteurs canadiens étatiques et non étatiques ne se positionnent pas sur l'ensemble de l'échiquier face à l'Europe d'aujourd'hui? Cette situation s'explique peut-être par le fait qu'au Canada les rapports entre le gouvernement et la société civile diffèrent de ceux aux États-Unis, ce qui se répercute au niveau international. Il existe aussi une grande variété d'acteurs issus de la société civile à Bruxelles, avec des objectifs, des intérêts et des thématiques de travail qui ne rejoignent pas nécessairement ceux poursuivis par les acteurs canadiens. C'est peut-être ce qui explique l'approche sélective adoptée par la Mission du Canada.

organise régulièrement des «Atlantic Rendez-vous» via satellite entre Bruxelles et Washington, regroupant des officiels et des experts européens et américains des enjeux abordés. Voir http://www.friendsofeurope.org/whatwedo.asp (page consultée le 1er mars 2007)

38 Security and Defence Agenda, What we do (En ligne), http://www.securitydefenceagenda.org/what_we_do.asp (page consultée le 27 février 2007)

${ }^{39}$ Forum Europe, About Us, (En ligne), http://www.forum-europe.com/about.asp (page consultée le 27 février 2007) 


\section{Conclusion}

En somme, la gestion de la sécurité est entrée dans un processus de transformation voire d'adaptation à la nouvelle donne internationale. Il n'est, dès lors, plus possible d'étudier l'élaboration de la PESC/PESD, les relations entre l'UE et le Canada et le positionnement du Canada en matière de politique étrangère et de sécurité, sans prendre en considération l'influence et l'intervention d'une grande variété d'acteurs publics et privés au niveau national, au niveau transnational et au niveau européen.

La gouvernance au sein de l'UE en matière de sécurité avec la PESC/PESD, avec tout ce qu'elle sous-tend, prend bien sûr une signification particulière en Europe en raison de l'intégration européenne. Pour certains, l'ouverture du processus décisionnel à des acteurs de la société civile vient démocratiser le système en plus de dépolitiser le processus. Un argument qui vient alimenter le débat sur la crise que traversent les démocraties à travers le monde. Pour d'autres, ce changement d'attitude représente plutôt un risque pour la démocratie, en déstabilisant le contrôle de l'État et en remettant en question les fondements mêmes de ses prérogatives. Le cas de la PESD est fort intéressant parce qu'il ouvre une brèche dans la gestion traditionnelle de la sécurité et que les paramètres de sa gestion viennent modifier la donne pour le Canada, un État tiers qui entretient des relations avec l'UE, mais également pour les acteurs de la société civile canadienne qui espèrent jouer un rôle sur la scène internationale; pourtant les deux restent en bout ligne encore en retrait dans cette nouvelle géométrie.

Dans un contexte, où certains n'hésitent pas à parler de la «bataille des idées » et de lutte de positionnement, où les interactions entre les différents acteurs qui participent au processus de dialogue qui précède les décisions de la PESD peuvent mener à des résultats concrets, je pense que la problématique soulevée dans cet article se pose et mérite d'être débattue pour comprendre comment les acteurs canadiens publics et privés évaluent l'UE et surtout, font connaître leur position. C'est toute la visibilité du Canada en Europe qui en découle. Si les acteurs canadiens étatiques et non étatiques semblent avoir pris acte de l'émergence de nouveaux acteurs publics et privés actifs au sein de l'UE, nous sommes à même de nous interroger : est-ce qu'ils en font suffisamment pour se positionner de façon stratégique en Europe? Encore faut-il savoir s'ils le souhaitent vraiment. Le débat est ouvert! 